\title{
Inventario y cartografía de focos de calor de Honduras, periodo 2000-2011, a partir de imágenes satelitales MODIS
}

\author{
José Jorge Escobar Figueroa, Nicolás Bladimir Castro Lagos
}

\section{RESUMEN}

Este estudio aborda el fenómeno natural de los incendios en Honduras, a partir del procesamiento de imágenes satelitales. El procedimiento comprende la obtención de las imágenes satelitales necesarias desde el servidor estándar de la NASA, las cuales se procesan cuidadosamente mediante un sistema de información geográfica, extrayendo la información pertinente para el análisis de periodicidad y la elaboración de la cartografía correspondiente.

Entre los resultados se logró obtener una tabla de datos, o inventario, con las cantidades de incendios y su localización en el territorio nacional durante un periodo de más de diez años; también se desarrolló la cartografía que corresponde a este inventario y el comportamiento en la periodicidad del fenómeno correspondiente a cada departamento del país.

Entre las conclusiones más significativas está la tendencia en la cantidad anual de los incendios en cada departamento de Honduras, además del periodo natural que presenta este fenómeno.

Palabras clave: MODIS, foco de calor, incendios, cartografía, sistema de información geográfica (SIG)

\section{ABSTRACT}

The object of study of this paper is the natural phenomenon of fires in Honduras. This is a first study of this natural phenomenon that encompasses the total national territory this satellite image processing. The method comprises obtaining the necessary satellite images from NASA standard server, these images are carefully processed in a Geographic Information System for extracting relevant frequency analysis and the preparation of the corresponding mapping information.

Among the findings it was possible to obtain a data table or inventory for the amount

\footnotetext{
${ }^{1}$ Universidad Nacional Autónoma de Honduras. Facultad de Ciencias, Instituto Hondureño de Ciencias de la Tierra.Correo electrónico: nicolascastro0@gmail.com
} 
of fire and its location in the country for over ten years, the mapping corresponding to this inventory is also developed further behavioral periodicity corresponding phenomenon each department of Honduras. As most significant findings is the trend in the annual number of fires in every department of the country, besides the natural period presented by this phenomenon.

Keywords: MODIS, focus heat, fires, cartography, GIS (Geographic Information System).

\section{INTRODUCCIÓN}

Un incendio forestal se puede definir como el fuego que se expande sin control. En el estudio de los incendios forestales, la teledetección o detección por satélite se ha constituido en una herramienta de gran potencial para suministrar información detallada y a gran escala de las superficies afectadas (Chuvieco Salinero, 2008). De esta forma, al evaluar la fase posterior al incendio forestal mediante la teledetección se trabajan dos etapas: discriminando zonas quemadas y la severidad de fuego, la primera es la finalidad de este trabajo.

El manejo de información y datos georreferenciados concernientes a incendios es importante porque no solamente da a conocer dónde ocurren los incendios, sino que posibilita cruzar estos datos con otro tipo de información georreferenciada, tales como la altura, población, actividad humana, etc., de forma que se pueden explicar con más veracidad las causas de los incendios y, posteriormente, elaborar métodos de prevención y control más eficaces.

No obstante, este trabajo no tiene como propósito hacer un cruce de información, ni explicar las causas o consecuencias de los incendios, sino dar un buen punto de partida para un trabajo de este tipo.

Para tales efectos, se elaboró la cartografía de los focos de calor por mes y año del territorio nacional correspondiente a doce años, de 2000 a 2011, para obtener la georreferenciación de estos focos de calor con una incertidumbre aceptable como un estudio preliminar a gran escala.

\section{METODOLOGÍA}

El sensor MODIS (moderate resolution imaging spectroradiometer) fue lanzado por la NASA a bordo del satélite Terra. Orbita la tierra a una altura de $705 \mathrm{~km}$. MODIS, Terra y Aqua cubren toda la superficie de la Tierra cada 102 días, generando datos 
en 36 bandas espectrales. Su objetivo es proveer series de observaciones globales de la atmósfera, los océanos y la Tierra, con resoluciones espaciales de entre $250 \mathrm{~m}$ y $1 \mathrm{~km}$. Las imágenes vienen en una proyección sinusoidal (monitoring global fires from EOS-MODIS).

Las técnicas de detección de incendios MODIS están automatizadas mediante un algoritmo que examina cada píxel de la imagen según la franja del espectro electromagnético, obteniendo un producto llamado MODIS MOD14A1, el cual asigna a cada píxel una categoría como: falta de datos, nubes, agua, no fuego, fuego, desconocido (algorithm technical background document).

Según la metodología utilizada, el producto MODIS MOD14A1 se descargó desde el servidor de la NASA llamado warehouse inventory search tool (actualmente este servidor fue reemplazado por el Reverb | ECHO), estos productos MODIS son compuestos de 8 días de incendios con píxel de $1 \mathrm{~km}$. Ya descargadas las imágenes se procede a realizar una reproyección a coordenadas UTM (huso 16) utilizando como elipsoide de referencia WGS84. Como los datos son obtenidos en formato HDF, son convertidos a formato IMG y luego exportados a un sistema de información geográfica. Se extrae de la imagen total solo a Honduras, separando de la imagen los pixeles de fuego.

Figura 1. Obtención, reproyección, recorte y extracción de las imágenes satelitales

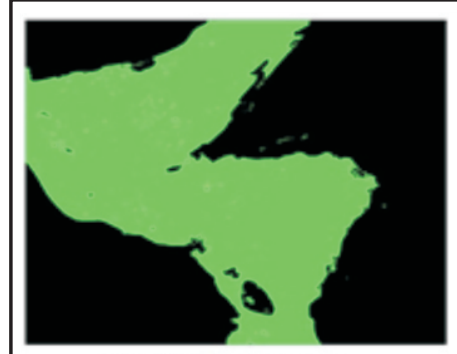

PROYECCION SINUSOIDAL

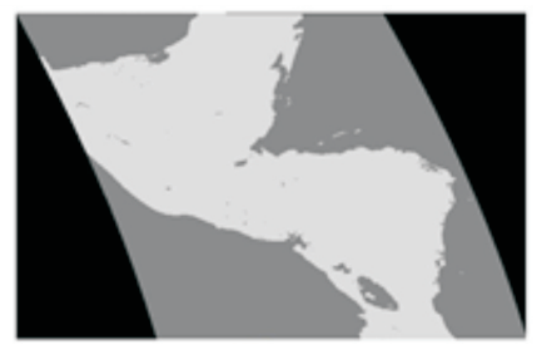

PROYECCION UTM WGS84

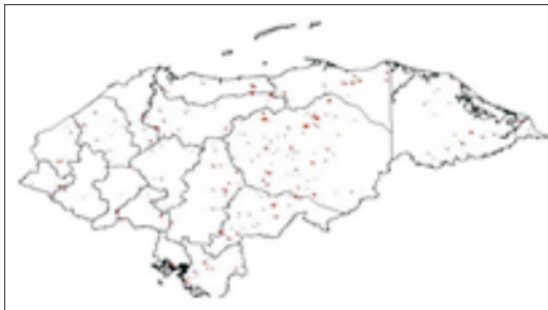

EXTRACTO DE LOS PIXELES FUEGO DEL TERRITORIO

Fuente: Elaboración propia con datos de la NASA 
Seguidamente, solo se tiene un conjunto de píxeles que corresponden a potenciales incendios, en el SIG se transforman a formato punto para ser procesados.

Figura 2. Fuego detectado, pixel clasificado por el algoritmo de MODIS, a punto para su procesamiento

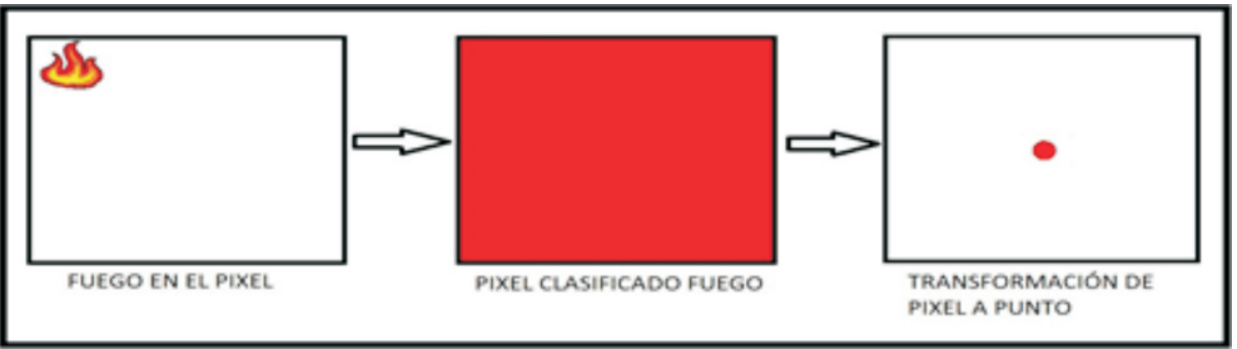

Luego, lo más apropiado es juntar y clasificar los datos en mensuales y anuales, ya que permite hacer una comparación temporal entre los meses de mayor y menor incidencia de incendios en un determinado año. Lo siguiente es agrupar por departamento los focos de calor e inventariar anualmente en tablas, las cuales se exportan con extensión .dat a un programa de procesamiento numérico, en donde es sometido a una rutina escrita para encontrar la tendencia en la cantidad y periodicidad en los incendios departamentales, utilizando un método de procesamiento de señales discretas llamado transformada de fourier finita. Por último, se elabora la cartografía de focos de calor para los doce años.

\section{ANÁLISIS DE LOS DATOS}

Los resultados se presentan en dos apartados: primero el inventario anual, luego el análisis de periodicidad y tendencia.

Inventario anual: en este se da el inventario de incendios anual de cada departamento en forma tabulada, además desarrolla la cartografía correspondiente a cada año, la cual no se presenta aquí por motivos de espacio. Es importante aclarar que los focos de calor o potenciales incendios son puntos georreferenciados, los cuales deben considerarse como el inventario en bruto. ${ }^{2}$

\footnotetext{
${ }^{2}$ Para obtener la cartografía y el inventario georreferenciado, contactar a los autores.
} 
Cuadro 1. Inventario año 2000

\begin{tabular}{|l|c|c|c|c|c|c|c|c|c|c|}
\hline \multirow{2}{*}{ Departamento } & \multicolumn{8}{|c|}{ Cantidad de focos de calor mensual } \\
\cline { 2 - 14 } & Mar. & Abr. & May. & Jun. & Jul. & Ago. & Sept. & Oct. & Nov. & Dic. \\
\hline Atlántida & 1 & 7 & 18 & 2 & 0 & 0 & 0 & 0 & 0 & 0 \\
\hline Choluteca & 38 & 67 & 10 & 0 & 0 & 4 & 0 & 6 & 11 & 11 \\
\hline Colón & 4 & 79 & 97 & 1 & 3 & 0 & 1 & 0 & 2 & 0 \\
\hline Comayagua & 10 & 51 & 8 & 0 & 0 & 0 & 0 & 0 & 2 & 0 \\
\hline Copán & 0 & 16 & 4 & 0 & 0 & 0 & 0 & 0 & 0 & 0 \\
\hline Cortés & 8 & 21 & 4 & 0 & 0 & 0 & 0 & 0 & 2 & 0 \\
\hline El Paraíso & 22 & 152 & 36 & 0 & 0 & 0 & 0 & 0 & 0 & 0 \\
\hline Francisco Morazán & 39 & 178 & 23 & 0 & 0 & 0 & 0 & 0 & 0 & 0 \\
\hline Gracias a Dios & 21 & 117 & 27 & 0 & 0 & 0 & 1 & 1 & 3 & 4 \\
\hline Intibucá & 0 & 12 & 1 & 0 & 1 & 0 & 0 & 0 & 0 & 0 \\
\hline Islas de la Bahía & 0 & 0 & 0 & 0 & 0 & 0 & 0 & 0 & 0 & 0 \\
\hline La Paz & 10 & 15 & 1 & 0 & 0 & 0 & 0 & 0 & 0 & 0 \\
\hline Lempira & 3 & 54 & 0 & 0 & 0 & 0 & 0 & 0 & 0 & 1 \\
\hline Ocotepeque & 0 & 2 & 0 & 0 & 0 & 0 & 0 & 0 & 0 & 0 \\
\hline Olancho & 38 & 449 & 157 & 0 & 0 & 0 & 0 & 0 & 0 & 0 \\
\hline Santa Barbara & 3 & 9 & 11 & 0 & 0 & 0 & 0 & 0 & 0 & 0 \\
\hline Valle & 0 & 2 & 4 & 0 & 0 & 0 & 0 & 0 & 0 & 0 \\
\hline Yoro & 28 & 91 & 56 & 1 & 0 & 0 & 2 & 1 & 4 & 0 \\
\hline
\end{tabular}

Fuente: Elaboración propia.

Cuadro 2. Inventario año 2001

\begin{tabular}{|c|c|c|c|c|c|c|c|c|c|c|c|c|}
\hline \multirow{2}{*}{ Departamento } & \multicolumn{12}{|c|}{ Cantidad de focos de calor mensual } \\
\hline & Ene. & Feb. & Mar. & Abr. & May. & Jun. & Jul. & Ago. & Sept. & Oct. & Nov. & Dic. \\
\hline Atlántida & 0 & 0 & 4 & 7 & 6 & 3 & 0 & 0 & 3 & 0 & 0 & 0 \\
\hline Choluteca & 17 & 11 & 38 & 97 & 11 & 0 & 3 & 0 & 0 & 0 & 1 & 11 \\
\hline Colón & 0 & 0 & 0 & 30 & 20 & 17 & 4 & 0 & 0 & 0 & 0 & 0 \\
\hline Comayagua & 1 & 0 & 18 & 76 & 26 & 3 & 2 & 3 & 0 & 2 & 0 & 0 \\
\hline Copán & 0 & 0 & 3 & 12 & 33 & 0 & 0 & 0 & 0 & 0 & 0 & 0 \\
\hline Cortés & 0 & 3 & 31 & 21 & 41 & 16 & 0 & 0 & 1 & 0 & 0 & 0 \\
\hline El Paraíso & 0 & 0 & 22 & 45 & 77 & 0 & 1 & 0 & 0 & 0 & 0 & 0 \\
\hline Francisco Morazán & 0 & 6 & 30 & 118 & 22 & 0 & 5 & 0 & 0 & 0 & 0 & 2 \\
\hline Gracias a Dios & 0 & 5 & 26 & 78 & 72 & 1 & 6 & 2 & 0 & 0 & 0 & 1 \\
\hline Intibucá & 0 & 3 & 0 & 16 & 7 & 0 & 0 & 0 & 0 & 0 & 0 & 0 \\
\hline Islas de la Bahía & 0 & 0 & 0 & 0 & 0 & 0 & 0 & 0 & 0 & 0 & 0 & 0 \\
\hline La Paz & 0 & 0 & 13 & 27 & 6 & 0 & 0 & 0 & 0 & 0 & 0 & 0 \\
\hline Lempira & 0 & 5 & 34 & 67 & 27 & 0 & 0 & 0 & 0 & 0 & 0 & 0 \\
\hline Ocotepeque & 0 & 0 & 5 & 16 & 2 & 0 & 0 & 0 & 0 & 0 & 0 & 0 \\
\hline Olancho & 0 & 0 & 22 & 176 & 160 & 1 & 4 & 0 & 0 & 0 & 1 & 0 \\
\hline Santa Barbara & 2 & 0 & 5 & 50 & 116 & 0 & 0 & 0 & 0 & 0 & 0 & 0 \\
\hline Valle & 4 & 6 & 5 & 23 & 0 & 0 & 0 & 0 & 0 & 0 & 0 & 0 \\
\hline Yoro & 1 & 0 & 20 & 123 & 91 & 0 & 3 & 1 & 0 & 0 & 0 & 0 \\
\hline
\end{tabular}

Fuente: Elaboración propia. 
Cuadro 3. Inventario año 2002

\begin{tabular}{|c|c|c|c|c|c|c|c|c|c|c|c|c|}
\hline \multirow{2}{*}{ Departamento } & \multicolumn{12}{|c|}{ Cantidad de focos de calor mensual } \\
\hline & Ene. & Feb. & Mar. & Abr. & May. & Jun & Jul. & Ago & Sept. & Oct. & Nov. & Dic. \\
\hline Atlántida & 0 & 0 & 0 & 0 & 27 & 2 & 1 & 2 & 1 & 1 & 0 & 0 \\
\hline Choluteca & 18 & 20 & 23 & 92 & 55 & 0 & 1 & 2 & 6 & 3 & 3 & 15 \\
\hline Colón & 0 & 0 & 0 & 41 & 79 & 6 & 0 & 0 & 9 & 2 & 0 & 4 \\
\hline Comayagua & 3 & 2 & 14 & 16 & 56 & 0 & 0 & 0 & 0 & 0 & 0 & 0 \\
\hline Copán & 0 & 0 & 0 & 7 & 27 & 0 & 0 & 2 & 0 & 0 & 0 & 0 \\
\hline Cortés & 3 & 0 & 12 & 22 & 47 & 2 & 1 & 1 & 0 & 0 & 0 & 0 \\
\hline El Paraíso & 2 & 7 & 3 & 58 & 77 & 0 & 0 & 3 & 0 & 0 & 0 & 2 \\
\hline Francisco Morazán & 3 & 0 & 17 & 64 & 118 & 0 & 0 & 1 & 0 & 0 & 0 & 0 \\
\hline Gracias a Dios & 1 & 5 & 9 & 59 & 94 & 0 & 3 & 5 & 8 & 0 & 21 & 0 \\
\hline Intibucá & 0 & 0 & 0 & 9 & 4 & 15 & 0 & 0 & 0 & 0 & 0 & 0 \\
\hline Islas de la Bahía & 0 & 0 & 0 & 0 & 0 & 0 & 0 & 0 & 0 & 0 & 0 & 0 \\
\hline LaPaz & 1 & 4 & 0 & 11 & 31 & 0 & 0 & 0 & 0 & 0 & 0 & 0 \\
\hline Lempira & 0 & 0 & 0 & 28 & 25 & 0 & 0 & 0 & 0 & 0 & 0 & 0 \\
\hline Ocotepeque & 0 & 0 & 0 & 9 & 5 & 0 & 0 & 0 & 0 & 0 & 0 & 0 \\
\hline Olancho & 0 & 3 & 2 & 167 & 230 & 0 & 0 & 0 & 0 & 3 & 0 & 0 \\
\hline Santa Barbara & 0 & 1 & 2 & 11 & 101 & 0 & 0 & 1 & 1 & 0 & 0 & 0 \\
\hline Valle & 1 & 2 & 0 & 14 & 11 & 0 & 0 & 1 & 0 & 3 & 4 & 0 \\
\hline Yoro & 2 & 0 & 6 & 26 & 177 & 0 & 1 & 0 & 0 & 1 & 0 & 0 \\
\hline
\end{tabular}

Fuente: Elaboración propia.

Cuadro 4. Inventario año 2003

\begin{tabular}{|c|c|c|c|c|c|c|c|c|c|c|c|c|}
\hline \multirow{2}{*}{ Departamento } & \multicolumn{12}{|c|}{ Cantidad de focos de calor mensual } \\
\hline & Ene. & Feb. & Mar. & Abr. & May. & Jun. & Jul. & Ago. & Sept. & Oct. & Nov. & Dic. \\
\hline Atlántida & 0 & 0 & 1 & 3 & 22 & 14 & 0 & 0 & 0 & 0 & 0 & 0 \\
\hline Choluteca & 7 & 13 & 22 & 35 & 16 & 0 & 1 & 0 & 0 & 3 & 2 & 8 \\
\hline Colón & 0 & 0 & 5 & 59 & 71 & 47 & 2 & 4 & 7 & 8 & 0 & 0 \\
\hline Comayagua & 0 & 11 & 22 & 52 & 36 & 1 & 0 & 0 & 2 & 0 & 0 & 4 \\
\hline Copán & 0 & 0 & 12 & 8 & 16 & 0 & 0 & 0 & 0 & 0 & 0 & 0 \\
\hline Cortés & 0 & 1 & 21 & 15 & 18 & 0 & 0 & 1 & 7 & 0 & 0 & 0 \\
\hline El Paraíso & 0 & 1 & 31 & 150 & 122 & 0 & 0 & 0 & 0 & 0 & 0 & 0 \\
\hline Francisco Morazán & 2 & 2 & 152 & 98 & 48 & 0 & 0 & 0 & 0 & 2 & 0 & 0 \\
\hline Gracias a Dios & 4 & 3 & 236 & 88 & 77 & 4 & 0 & 0 & 0 & 0 & 1 & 2 \\
\hline Intibucá & 0 & 0 & 10 & 4 & 2 & 0 & 0 & 0 & 2 & 0 & 0 & 0 \\
\hline Islas de la Bahía & 0 & 0 & 0 & 1 & 0 & 0 & 0 & 0 & 0 & 0 & 0 & 0 \\
\hline La Paz & 0 & 2 & 9 & 2 & 3 & 0 & 0 & 0 & 0 & 0 & 0 & 0 \\
\hline Lempira & 0 & 0 & 9 & 27 & 15 & 0 & 0 & 0 & 0 & 0 & 0 & 0 \\
\hline Ocotepeque & 0 & 0 & 8 & 3 & 0 & 0 & 0 & 0 & 0 & 0 & 0 & 0 \\
\hline Olancho & 0 & 1 & 131 & 434 & 345 & 1 & 0 & 0 & 0 & 0 & 0 & 0 \\
\hline Santa Barbara & 0 & 1 & 10 & 38 & 102 & 1 & 0 & 0 & 0 & 0 & 0 & 0 \\
\hline Valle & 1 & 1 & 0 & 5 & 1 & 0 & 0 & 2 & 0 & 1 & 5 & 1 \\
\hline Yoro & 0 & 2 & 21 & 95 & 77 & 13 & 1 & 0 & 0 & 0 & 0 & 2 \\
\hline
\end{tabular}

Fuente: Elaboración propia. 
Cuadro 5. Inventario año 2004

\begin{tabular}{|l|c|c|c|c|c|c|c|c|c|c|c|c|c|}
\hline \multirow{2}{*}{ Departamento } & \multicolumn{10}{c|}{ Cantidad de focos de calor mensual } \\
\cline { 2 - 6 } & Ene. & Feb. & Mar. & Abr. & May. & Jun. & Jul. & Ago. & Sept. & Oct. & Nov. & Dic. \\
\hline Atlántida & 0 & 0 & 0 & 0 & 0 & 1 & 0 & 0 & 0 & 1 & 0 & 0 \\
\hline Choluteca & 25 & 18 & 49 & 82 & 38 & 0 & 0 & 4 & 5 & 0 & 4 & 19 \\
\hline Colón & 0 & 1 & 0 & 2 & 6 & 8 & 3 & 5 & 5 & 4 & 1 & 0 \\
\hline Comayagua & 0 & 4 & 1 & 10 & 0 & 0 & 5 & 0 & 3 & 0 & 2 & 1 \\
\hline Copán & 0 & 0 & 0 & 9 & 5 & 0 & 0 & 0 & 0 & 0 & 0 & 0 \\
\hline Cortés & 0 & 8 & 7 & 13 & 10 & 3 & 1 & 1 & 3 & 2 & 0 & 0 \\
\hline El Paraíso & 1 & 9 & 0 & 81 & 2 & 0 & 0 & 0 & 0 & 0 & 0 & 0 \\
\hline Francisco Morazán & 1 & 6 & 0 & 35 & 5 & 0 & 0 & 1 & 1 & 0 & 0 & 1 \\
\hline Gracias a Dios & 7 & 7 & 22 & 29 & 7 & 2 & 2 & 13 & 7 & 1 & 1 & 1 \\
\hline Intibucá & 0 & 0 & 2 & 4 & 1 & 2 & 0 & 0 & 0 & 0 & 0 & 0 \\
\hline Islas de la Bahía & 0 & 0 & 0 & 0 & 0 & 0 & 0 & 0 & 0 & 0 & 0 & 0 \\
\hline La Paz & 3 & 0 & 2 & 7 & 0 & 1 & 0 & 0 & 0 & 0 & 0 & 0 \\
\hline Lempira & 0 & 0 & 12 & 10 & 3 & 0 & 0 & 0 & 0 & 0 & 0 & 0 \\
\hline Ocotepeque & 0 & 0 & 1 & 15 & 0 & 0 & 0 & 0 & 0 & 0 & 0 & 0 \\
\hline Olancho & 0 & 0 & 9 & 22 & 2 & 1 & 0 & 2 & 3 & 2 & 0 & 0 \\
\hline Santa Barbara & 0 & 0 & 0 & 12 & 20 & 0 & 1 & 0 & 0 & 0 & 0 & 0 \\
\hline Valle & 2 & 1 & 4 & 10 & 8 & 0 & 0 & 0 & 2 & 3 & 3 & 0 \\
\hline Yoro & 0 & 3 & 1 & 31 & 12 & 2 & 4 & 0 & 0 & 1 & 2 & 0 \\
\hline
\end{tabular}

Fuente: Elaboración propia.

Cuadro 6. Inventario año 2005

\begin{tabular}{|c|c|c|c|c|c|c|c|c|c|c|c|c|}
\hline \multirow{2}{*}{ Departamento } & \multicolumn{12}{|c|}{ Cantidad de focos de calor mensual } \\
\hline & Ene. & Feb. & Mar. & Abr. & May. & Jun. & Jul. & Ago. & Sept. & Oct. & Nov. & Dic. \\
\hline Atlántida & 0 & 2 & 1 & 4 & 2 & 3 & 0 & 0 & 0 & 0 & 0 & 0 \\
\hline Choluteca & 23 & 30 & 18 & 44 & 3 & 0 & 0 & 0 & 0 & 1 & 3 & 4 \\
\hline Colón & 0 & 0 & 1 & 41 & 59 & 1 & 1 & 1 & 0 & 0 & 1 & 0 \\
\hline Comayagua & 1 & 2 & 48 & 113 & 2 & 0 & 0 & 0 & 1 & 0 & 0 & 1 \\
\hline Copán & 0 & 0 & 1 & 26 & 10 & 0 & 0 & 0 & 0 & 0 & 0 & 0 \\
\hline Cortés & 0 & 6 & 15 & 56 & 21 & 1 & 0 & 0 & 0 & 0 & 0 & 0 \\
\hline El Paraíso & 0 & 3 & 23 & 239 & 42 & 0 & 0 & 0 & 0 & 0 & 0 & 2 \\
\hline Francisco Morazán & 0 & 15 & 52 & 121 & 11 & 0 & 0 & 1 & 0 & 0 & 0 & 0 \\
\hline Gracias a Dios & 7 & 60 & 218 & 274 & 54 & 1 & 0 & 0 & 0 & 0 & 0 & 0 \\
\hline Intibucá & 0 & 2 & 1 & 3 & 9 & 0 & 0 & 0 & 0 & 0 & 0 & 0 \\
\hline Islas de la Bahía & 0 & 0 & 0 & 0 & 2 & 0 & 0 & 0 & 0 & 0 & 0 & 0 \\
\hline La Paz & 0 & 6 & 2 & 9 & 0 & 0 & 0 & 0 & 0 & 0 & 0 & 0 \\
\hline Lempira & 0 & 0 & 3 & 39 & 8 & 0 & 0 & 0 & 0 & 0 & 0 & 0 \\
\hline Ocotepeque & 0 & 1 & 0 & 4 & 0 & 0 & 0 & 0 & 0 & 0 & 0 & 0 \\
\hline Olancho & 0 & 7 & 102 & 750 & 159 & 0 & 2 & 1 & 0 & 0 & 0 & 0 \\
\hline Santa Barbara & 0 & 0 & 8 & 46 & 29 & 4 & 0 & 0 & 0 & 0 & 0 & 0 \\
\hline Valle & 0 & 6 & 2 & 5 & 0 & 0 & 0 & 0 & 2 & 0 & 1 & 0 \\
\hline Yoro & 0 & 13 & 90 & 251 & 42 & 1 & 0 & 0 & 0 & 0 & 0 & 0 \\
\hline
\end{tabular}

Fuente: Elaboración propia. 
Cuadro 7. Inventario año 2006

\begin{tabular}{|c|c|c|c|c|c|c|c|c|c|c|c|c|}
\hline \multirow{2}{*}{ Departamento } & \multicolumn{12}{|c|}{ Cantidad de focos de calor mensual } \\
\hline & Ene. & Feb & Mar. & Abr & May. & Jun. & Jul. & Ago. & Sept & Oct. & Nov. & Dic. \\
\hline Atlántida & 0 & 0 & 0 & 3 & 36 & 0 & 0 & 3 & 0 & 0 & 0 & 0 \\
\hline Choluteca & 11 & 12 & 67 & 66 & 10 & 0 & 0 & 1 & 0 & 1 & 2 & 9 \\
\hline Colón & 0 & 0 & 12 & 16 & 72 & 8 & 1 & 1 & 7 & 1 & 0 & 0 \\
\hline Comayagua & 0 & 2 & 18 & 34 & 20 & 0 & 0 & 0 & 1 & 0 & 0 & 0 \\
\hline Copán & 0 & 0 & 4 & 5 & 13 & 0 & 0 & 0 & 0 & 0 & 0 & 0 \\
\hline Cortés & 0 & 2 & 14 & 17 & 17 & 0 & 0 & 0 & 2 & 2 & 0 & 0 \\
\hline El Paraíso & 2 & 1 & 9 & 52 & 58 & 5 & 0 & 0 & 0 & 0 & 0 & 0 \\
\hline Francisco Morazán & 0 & 1 & 54 & 69 & 52 & 3 & 0 & 0 & 2 & 0 & 0 & 0 \\
\hline Gracias a Dios & 0 & 0 & 34 & 48 & 63 & 3 & 1 & 0 & 1 & 1 & 6 & 0 \\
\hline Intibucá & 0 & 0 & 2 & 1 & 4 & 0 & 0 & 0 & 0 & 0 & 0 & 0 \\
\hline Islas de la Bahía & 0 & 0 & 0 & 0 & 0 & 0 & 0 & 0 & 0 & 0 & 0 & 0 \\
\hline La Paz & 0 & 4 & 7 & 18 & 3 & 0 & 0 & 0 & 0 & 0 & 0 & 0 \\
\hline Lempira & 0 & 1 & 6 & 21 & 6 & 0 & 0 & 0 & 0 & 0 & 0 & 0 \\
\hline Ocotepeque & 0 & 0 & 6 & 13 & 3 & 0 & 0 & 0 & 0 & 0 & 0 & 0 \\
\hline Olancho & 0 & 0 & 7 & 166 & 235 & 13 & 0 & 0 & 0 & 0 & 0 & 0 \\
\hline Santa Barbara & 0 & 0 & 4 & 20 & 44 & 0 & 0 & 0 & 0 & 0 & 0 & 0 \\
\hline Valle & 0 & 4 & 12 & 3 & 4 & 1 & 1 & 1 & 7 & 5 & 1 & 2 \\
\hline Yoro & 0 & 0 & 11 & 54 & 94 & 2 & 1 & 1 & 0 & 0 & 0 & 0 \\
\hline
\end{tabular}

Fuente: Elaboración propia.

Cuadro 8. Inventario año 2007

\begin{tabular}{|c|c|c|c|c|c|c|c|c|c|c|c|c|}
\hline \multirow{2}{*}{ Departamento } & \multicolumn{12}{|c|}{ Cantidad de focos de calor mensual } \\
\hline & Ene. & Feb. & Mar. & Abr. & May. & Jun. & Jul. & Ago. & Sept. & Oct. & Nov. & Dic. \\
\hline Atlántida & 0 & 0 & 0 & 3 & 9 & 0 & 0 & 0 & 0 & 0 & 0 & 0 \\
\hline Choluteca & 17 & 19 & 28 & 27 & 3 & 1 & 0 & 0 & 0 & 5 & 2 & 5 \\
\hline Colón & 0 & 2 & 0 & 7 & 29 & 5 & 1 & 4 & 6 & 1 & 0 & 0 \\
\hline Comayagua & 0 & 1 & 12 & 17 & 31 & 1 & 1 & 0 & 0 & 1 & 0 & 0 \\
\hline Copán & 0 & 0 & 0 & 19 & 27 & 0 & 0 & 0 & 0 & 0 & 0 & 0 \\
\hline Cortés & 4 & 4 & 6 & 12 & 49 & 3 & 0 & 0 & 0 & 2 & 0 & 0 \\
\hline El Paraíso & 1 & 6 & 7 & 15 & 29 & 0 & 0 & 2 & 0 & 8 & 0 & 0 \\
\hline Francisco Morazán & 0 & 6 & 6 & 19 & 16 & 3 & 0 & 0 & 0 & 0 & 0 & 0 \\
\hline Gracias a Dios & 4 & 27 & 39 & 30 & 35 & 2 & 0 & 1 & 1 & 17 & 2 & 6 \\
\hline Intibucá & 0 & 0 & 8 & 5 & 12 & 0 & 0 & 0 & 0 & 0 & 0 & 0 \\
\hline Islas de la Bahía & 0 & 0 & 0 & 0 & 0 & 0 & 0 & 0 & 0 & 0 & 0 & 0 \\
\hline La Paz & 0 & 0 & 4 & 6 & 4 & 0 & 0 & 0 & 0 & 0 & 0 & 1 \\
\hline Lempira & 0 & 0 & 10 & 8 & 20 & 0 & 0 & 0 & 0 & 0 & 0 & 0 \\
\hline Ocotepeque & 0 & 0 & 1 & 3 & 8 & 0 & 0 & 0 & 0 & 0 & 2 & 0 \\
\hline Olancho & 0 & 0 & 3 & 40 & 168 & 0 & 0 & 0 & 2 & 1 & 0 & 0 \\
\hline Santa Barbara & 0 & 0 & 2 & 25 & 148 & 0 & 1 & 0 & 0 & 0 & 1 & 1 \\
\hline Valle & 2 & 4 & 3 & 0 & 3 & 0 & 0 & 1 & 2 & 1 & 2 & 0 \\
\hline Yoro & 0 & 5 & 8 & 46 & 121 & 13 & 0 & 0 & 0 & 0 & 0 & 0 \\
\hline
\end{tabular}

Fuente: Elaboración propia. 
Cuadro 9. Inventario año 2008

\section{Departamento}

Cantidad de focos de calor mensual

Atlántida \begin{tabular}{|l|l|l|l|l|l|l|}
\hline Ene. & Feb. & Mar. & Abr. May. Jun. Jul. Ago. Sept. Oct. & Nov. & Dic. \\
\hline
\end{tabular}

Choluteca \begin{tabular}{l|l|l|c|c|}
0 & 0 & 0 & 9 & 13 \\
0 & 5 & 7 & 25 & 65 \\
\hline
\end{tabular}

Colón

10

$19 \quad 20$

Comayagua

\begin{tabular}{l|l|l|l|l|l}
0 & 2 & 1 & 19 & -1 \\
0 & 0 & 0 & 2
\end{tabular}

Copán \begin{tabular}{l|l|l|l|l}
11 & 2 & 6 & 9 & 5
\end{tabular}

Cortés $\begin{array}{lll}0 & 5 & 15\end{array}$

El Paraíso

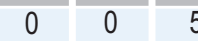

Francisco Morazán

Gracias a Dios

Intibucá

Islas de la Bahía

\begin{tabular}{l|l|l|l|l|l}
0 & 4 & 8 & 101 & 86 & 0 \\
0 & 3 & 38 & 48 & 108 & 8
\end{tabular}

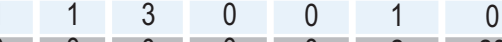

$61 \quad 20$

$17-10000$

$\begin{array}{lll}0 & 0 & 0\end{array}$

0

La Paz

Lempira

\begin{tabular}{l|c|c|c|c|c|c|}
0 & 3 & 38 & 48 & 108 & 8 \\
0 & 0 & 2 & 4 & 0 & 0 & 0
\end{tabular}

\begin{tabular}{|l|l|l|l|l|l|l|l|l|l|l|l|l|}
\hline Ocotepeque & 0 & 0 & 0 & 4 & 0 & 0 & 0 & 0 & 0 & 0 & 0 & 0 \\
\hline Olancho & 0 & 3 & 4 & 104 & 292 & 1 & 0 & 0 & 2 & 0 & 0 & 0 \\
\hline
\end{tabular}

$\begin{array}{lllllllllllll}0 & 0 & 2 & 4 & 0 & 0 & 0 & 0 & 0 & 0 & 0 & 0\end{array}$

\begin{tabular}{l|l|l|l|l|l|l|l|l|l|l|l|l|l|} 
Santa Barbara & 1 & 2 & 0 & 6 & 83 & 0 & 0 & 0 & 0 & 0 & 0 & 0
\end{tabular}

Valle

Yoro

\begin{tabular}{l|l|l|l|l|l|l|}
2 & 0 & 6 & 7 & 0 \\
\hline & 5 & 2 & 22 & 95 & 5 \\
\hline
\end{tabular}

\begin{tabular}{ll|l|l|l}
2 & 0 & 6 & 7 & 0 \\
1 & 5 & 2 & 22 & 95
\end{tabular}

$0 \quad 0$

Fuente: Elaboración propia.

Cuadro 10. Inventario año 2009

\begin{tabular}{|c|c|c|c|c|c|c|c|c|c|c|c|c|}
\hline \multirow{2}{*}{ Departamento } & \multicolumn{12}{|c|}{ Cantidad de focos de calor mensual } \\
\hline & Ene. & Feb. & Mar. & Abr. & May. & Jun. & Jul. & Ago. & Sept. & Oct. & Nov. & Dic. \\
\hline Atlántida & 0 & 0 & 1 & 6 & 16 & 2 & 0 & 0 & 0 & 0 & 0 & 0 \\
\hline Choluteca & 28 & 17 & 24 & 90 & 7 & 2 & 0 & 1 & 2 & 7 & 3 & 24 \\
\hline Colón & 0 & 0 & 3 & 23 & 52 & 11 & 2 & 3 & 6 & 6 & 1 & 4 \\
\hline Comayagua & 0 & 1 & 12 & 73 & 5 & 2 & 0 & 0 & 1 & 0 & 0 & 0 \\
\hline Copán & 0 & 0 & 1 & 25 & 1 & 0 & 0 & 0 & 0 & 0 & 0 & 0 \\
\hline Cortés & 0 & 0 & 18 & 14 & 14 & 5 & 2 & 4 & 0 & 1 & 1 & 0 \\
\hline El Paraíso & 0 & 0 & 15 & 115 & 5 & 0 & 0 & 0 & 2 & 1 & 0 & 0 \\
\hline Francisco Morazán & 2 & 1 & 33 & 212 & 6 & 0 & 2 & 0 & 2 & 1 & 0 & 0 \\
\hline Gracias a Dios & 5 & 6 & 32 & 130 & 6 & 0 & 7 & 0 & 4 & 1 & 5 & 9 \\
\hline Intibucá & 0 & 0 & 3 & 130 & 0 & 0 & 0 & 0 & 0 & 0 & 0 & 0 \\
\hline Islas de la Bahía & 0 & 0 & 0 & 0 & 0 & 0 & 0 & 0 & 0 & 0 & 0 & 0 \\
\hline La Paz & 0 & 0 & 7 & 20 & 0 & 0 & 0 & 0 & 0 & 0 & 0 & 0 \\
\hline Lempira & 5 & 0 & 9 & 24 & 0 & 0 & 0 & 0 & 0 & 0 & 0 & 0 \\
\hline Ocotepeque & 0 & 0 & 0 & 4 & 0 & 0 & 0 & 0 & 0 & 0 & 0 & 0 \\
\hline Olancho & 0 & 0 & 23 & 228 & 10 & 0 & 0 & 0 & 2 & 2 & 0 & 0 \\
\hline Santa Barbara & 0 & 0 & 3 & 21 & 33 & 9 & 0 & 0 & 5 & 0 & 0 & 0 \\
\hline Valle & 3 & 6 & 2 & 9 & 3 & 1 & 0 & 6 & 3 & 4 & 2 & 1 \\
\hline Yoro & 0 & 3 & 5 & 78 & 30 & 8 & 0 & 0 & 0 & 0 & 0 & 0 \\
\hline
\end{tabular}

Fuente: Elaboración propia. 
Cuadro 11. Inventario año 2010

\begin{tabular}{|c|c|c|c|c|c|c|c|c|c|c|c|c|}
\hline \multirow{2}{*}{ Departamento } & \multicolumn{12}{|c|}{ Cantidad de focos de calor mensual } \\
\hline & Ene. & Feb. & Mar. & Abr. & May. & Jun. & Jul. & Ago. & Sept. & Oct. & Nov. & Dic. \\
\hline Atlántida & 0 & 1 & 0 & 3 & 3 & 4 & 0 & 0 & 0 & 0 & 0 & 0 \\
\hline Choluteca & 35 & 15 & 37 & 37 & 2 & 0 & 0 & 2 & 1 & 2 & 14 & 4 \\
\hline Colón & 0 & 0 & 1 & 18 & 5 & 16 & 1 & 0 & 10 & 0 & 2 & 0 \\
\hline Comayagua & 0 & 0 & 8 & 9 & 5 & 0 & 0 & 0 & 0 & 0 & 0 & 1 \\
\hline Copán & 0 & 0 & 1 & 6 & 0 & 0 & 0 & 0 & 1 & 0 & 0 & 0 \\
\hline Cortés & 1 & 10 & 20 & 28 & 1 & 1 & 1 & 9 & 8 & 0 & 7 & 0 \\
\hline El Paraíso & 1 & 6 & 29 & 21 & 1 & 0 & 0 & 0 & 0 & 0 & 1 & 0 \\
\hline Francisco Morazán & 9 & 2 & 48 & 11 & 0 & 0 & 0 & 0 & 0 & 0 & 0 & 0 \\
\hline Gracias a Dios & 14 & 25 & 84 & 23 & 14 & 2 & 3 & 0 & 6 & 0 & 12 & 0 \\
\hline Intibucá & 0 & 1 & 0 & 1 & 1 & 0 & 0 & 0 & 0 & 0 & 0 & 0 \\
\hline Islas de la Bahía & 0 & 0 & 0 & 0 & 0 & 0 & 0 & 0 & 0 & 0 & 0 & 0 \\
\hline La Paz & 0 & 0 & 1 & 1 & 2 & 0 & 0 & 0 & 0 & 0 & 0 & 0 \\
\hline Lempira & 0 & 0 & 10 & 10 & 3 & 0 & 0 & 0 & 0 & 0 & 0 & 0 \\
\hline Ocotepeque & 0 & 0 & 0 & 3 & 0 & 0 & 0 & 0 & 0 & 0 & 0 & 0 \\
\hline Olancho & 0 & 1 & 38 & 37 & 19 & 2 & 1 & 1 & 5 & 0 & 0 & 0 \\
\hline Santa Barbara & 0 & 0 & 8 & 14 & 3 & 7 & 0 & 0 & 1 & 0 & 0 & 0 \\
\hline Valle & 1 & 5 & 0 & 3 & 0 & 0 & 0 & 0 & 0 & 0 & 0 & 0 \\
\hline Yoro & 5 & 0 & 4 & 25 & 2 & 0 & 0 & 0 & 1 & 0 & 0 & 0 \\
\hline
\end{tabular}

Fuente: Elaboración propia.

Cuadro 12. Inventario año 2011

\begin{tabular}{|c|c|c|c|c|c|c|c|c|c|c|c|c|}
\hline \multirow{2}{*}{ Departamento } & \multicolumn{12}{|c|}{ Cantidad de focos de calor mensual } \\
\hline & Ene. & Feb. & Mar. & Abr. & May. & Jun. & Jul. & Ago. & Sept. & Oct. & Nov. & Dic. \\
\hline Atlántida & 0 & 0 & 1 & 15 & 3 & 2 & 0 & 0 & 0 & 0 & 0 & 0 \\
\hline Choluteca & 0 & 0 & 3 & 217 & 108 & 9 & 3 & 4 & 4 & 1 & 0 & 0 \\
\hline Colón & 3 & 0 & 27 & 73 & 5 & 0 & 0 & 5 & 2 & 0 & 0 & 0 \\
\hline Comayagua & 0 & 0 & 6 & 14 & 10 & 0 & 0 & 3 & 2 & 0 & 0 & 0 \\
\hline Copán & 3 & 4 & 10 & 60 & 27 & 9 & 2 & 1 & 3 & 0 & 1 & 0 \\
\hline Cortés & 13 & 23 & 23 & 79 & 20 & 0 & 0 & 2 & 0 & 0 & 9 & 12 \\
\hline El Paraíso & 4 & 0 & 65 & 236 & 26 & 1 & 0 & 0 & 0 & 0 & 1 & 1 \\
\hline Francisco Morazán & 1 & 2 & 64 & 159 & 13 & 0 & 0 & 0 & 0 & 0 & 0 & 1 \\
\hline Gracias a Dios & 4 & 0 & 15 & 129 & 44 & 0 & 0 & 3 & 1 & 1 & 13 & 3 \\
\hline Intibucá & 0 & 0 & 0 & 12 & 6 & 0 & 0 & 0 & 0 & 0 & 0 & 0 \\
\hline Islas de la Bahía & 0 & 0 & 0 & 0 & 0 & 0 & 0 & 0 & 0 & 0 & 0 & 0 \\
\hline La Paz & 0 & 3 & 6 & 18 & 1 & 0 & 0 & 0 & 0 & 0 & 0 & 0 \\
\hline Lempira & 0 & 6 & 12 & 16 & 4 & 0 & 0 & 0 & 0 & 0 & 0 & 0 \\
\hline Ocotepeque & 0 & 0 & 0 & 4 & 5 & 0 & 0 & 0 & 0 & 0 & 0 & 0 \\
\hline Olancho & 1 & 4 & 85 & 737 & 109 & 1 & 0 & 1 & 1 & 0 & 0 & 0 \\
\hline Santa Barbara & 0 & 2 & 6 & 44 & 49 & 3 & 0 & 0 & 0 & 0 & 0 & 0 \\
\hline Valle & 0 & 0 & 1 & 6 & 6 & 0 & 0 & 0 & 0 & 0 & 3 & 5 \\
\hline Yoro & 0 & 4 & 15 & 255 & 69 & 2 & 0 & 3 & 1 & 0 & 0 & 0 \\
\hline
\end{tabular}

Fuente: Elaboración propia. 
En el cuadro 13 se presentan los datos correspondientes a los totales anuales por cada departamento, en esta se puede observar qué departamento tuvo más incidencia de incendios $y$, debido a que las áreas de cada departamento son diferentes, se muestran los incendios, por metro cuadrado, ocurridos en los doce años.

Seguidamente, en el mapa 1, se muestra la composición de incendios de los doce años, en el cual se ve el patrón de distribución geográfica de los incendios en el territorio.

\section{Cuadro 13. Inventario año 2012}

\begin{tabular}{|c|c|c|c|c|c|c|c|c|c|c|c|c|c|c|}
\hline \multirow{2}{*}{ Depto. } & \multicolumn{13}{|c|}{ Años } & \multirow{2}{*}{$\begin{array}{l}\text { Incedios por } \\
\text { cada } 10 \mathrm{~km}^{2}\end{array}$} \\
\hline & 2000 & 2001 & 2002 & 2003 & 2004 & 2005 & 2006 & 2007 & 2008 & 2009 & 2010 & 2011 & Total & \\
\hline Atlántida & 28 & 23 & 34 & 40 & 2 & 12 & 72 & 12 & 23 & 25 & 11 & 21 & 273 & 0.6 \\
\hline Choluteca & 147 & 189 & 238 & 107 & 244 & 126 & 179 & 107 & 134 & 205 & 149 & 181 & 2006 & 4.6 \\
\hline Colón & 187 & 71 & 141 & 203 & 35 & 105 & 118 & 55 & 115 & 111 & 53 & 349 & 1543 & 1.9 \\
\hline Comayagua & 71 & 131 & 91 & 128 & 26 & 168 & 75 & 64 & 48 & 94 & 23 & 115 & 1034 & 2.0 \\
\hline Copán & 20 & 48 & 38 & 36 & 14 & 37 & 22 & 46 & 22 & 27 & 8 & 35 & 353 & 1.1 \\
\hline Cortés & 35 & 113 & 86 & 63 & 48 & 99 & 54 & 80 & 77 & 59 & 86 & 120 & 920 & 2.4 \\
\hline El Paraíso & 210 & 145 & 152 & 304 & 93 & 309 & 127 & 68 & 133 & 138 & 59 & 334 & 2072 & 2.8 \\
\hline Fco. Morazán & 240 & 183 & 203 & 304 & 50 & 200 & 181 & 50 & 199 & 259 & 70 & 240 & 2179 & 2.6 \\
\hline Gracias a Dios & 174 & 191 & 220 & 415 & 99 & 614 & 157 & 164 & 215 & 205 & 183 & 213 & 2850 & 1.8 \\
\hline Intibucá & 14 & 26 & 13 & 18 & 9 & 15 & 7 & 25 & 6 & 16 & 3 & 18 & 170 & 0.5 \\
\hline Islas de laBahía & 0 & 0 & 0 & 1 & 0 & 2 & 0 & 0 & 0 & 0 & 0 & 0 & 3 & 0.1 \\
\hline LaPaz & 26 & 46 & 47 & 16 & 13 & 17 & 32 & 15 & 3 & 27 & 4 & 28 & 274 & 1.1 \\
\hline Lempira & 64 & 133 & 53 & 51 & 25 & 50 & 34 & 38 & 36 & 38 & 23 & 38 & 583 & 1.4 \\
\hline Ocotepeque & 2 & 23 & 14 & 11 & 16 & 5 & 22 & 14 & 4 & 4 & 3 & 9 & 127 & 0.8 \\
\hline Olancho & 644 & 364 & 405 & 912 & 41 & 1021 & 421 & 214 & 406 & 265 & 104 & 939 & 5736 & 2.4 \\
\hline Santa Barbara & 23 & 173 & 117 & 152 & 33 & 87 & 68 & 178 & 92 & 71 & 33 & 104 & 1131 & 2.2 \\
\hline Valle & 6 & 38 & 36 & 17 & 33 & 16 & 41 & 18 & 18 & 40 & 9 & 21 & 293 & 1.8 \\
\hline Yoro & 183 & 239 & 213 & 211 & 56 & 397 & 163 & 193 & 131 & 124 & 37 & 349 & 2296 & 3.0 \\
\hline Total & 2074 & 2136 & 2101 & 2989 & 837 & 3280 & 1743 & 1341 & 1662 & 1708 & 858 & 3114 & 23843 & \\
\hline
\end{tabular}

Fuente: Elaboración propia.

En la tabla 13 aparecen los años 2005 y 2011 como los que tuvieron más incendios; los años anteriores a estos, 2004 y 2010, tuvieron menos incidencia. Olancho tuvo más incendios, pero Choluteca tiene más área quemada.

Entre abril mayo y marzo está el $90 \%$ de los incendios anuales. 
Gráfico 1. Porcentaje de incendios por departamento, 2000-2011

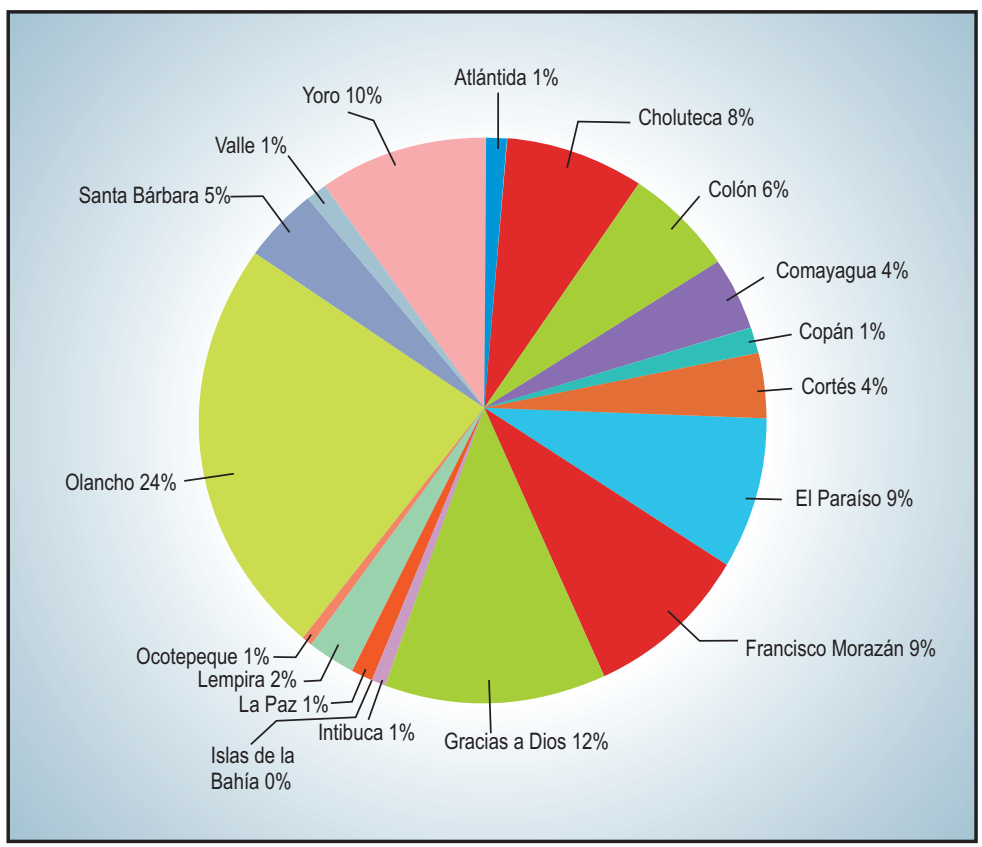

Gráfico 2. Porcentaje de incendios mensuales, 2000-2011

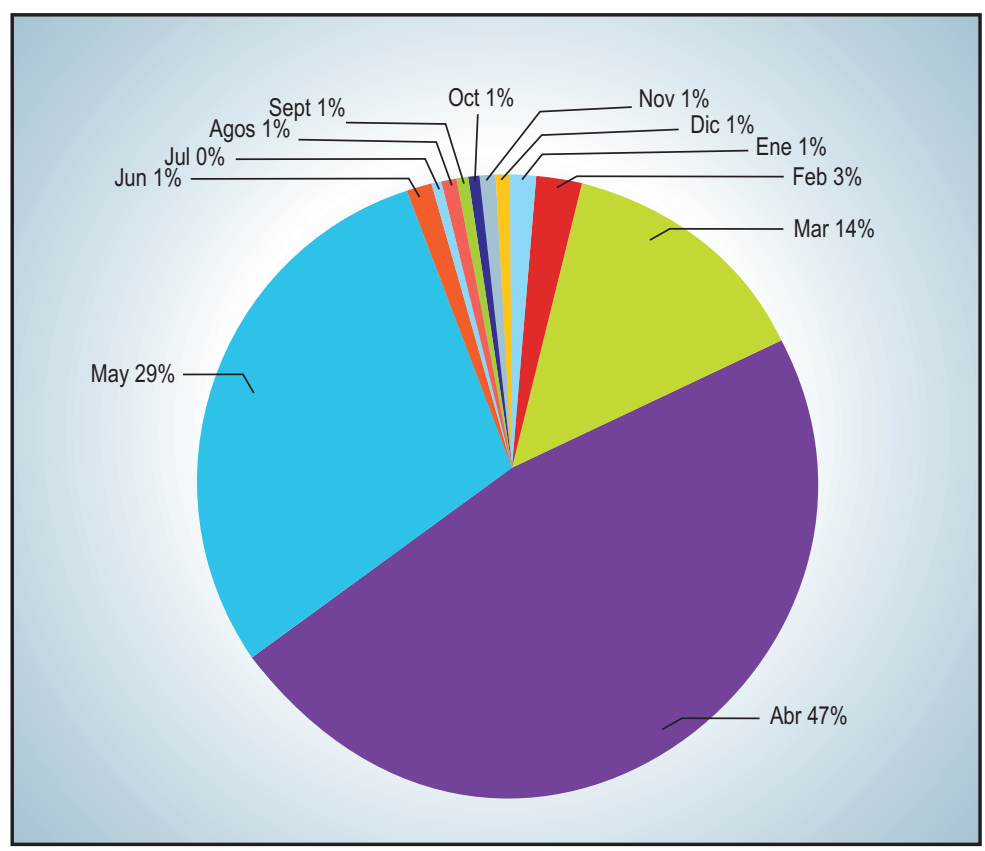


Mapa 1. Distribución geográfica de los incendios, 2000-2011

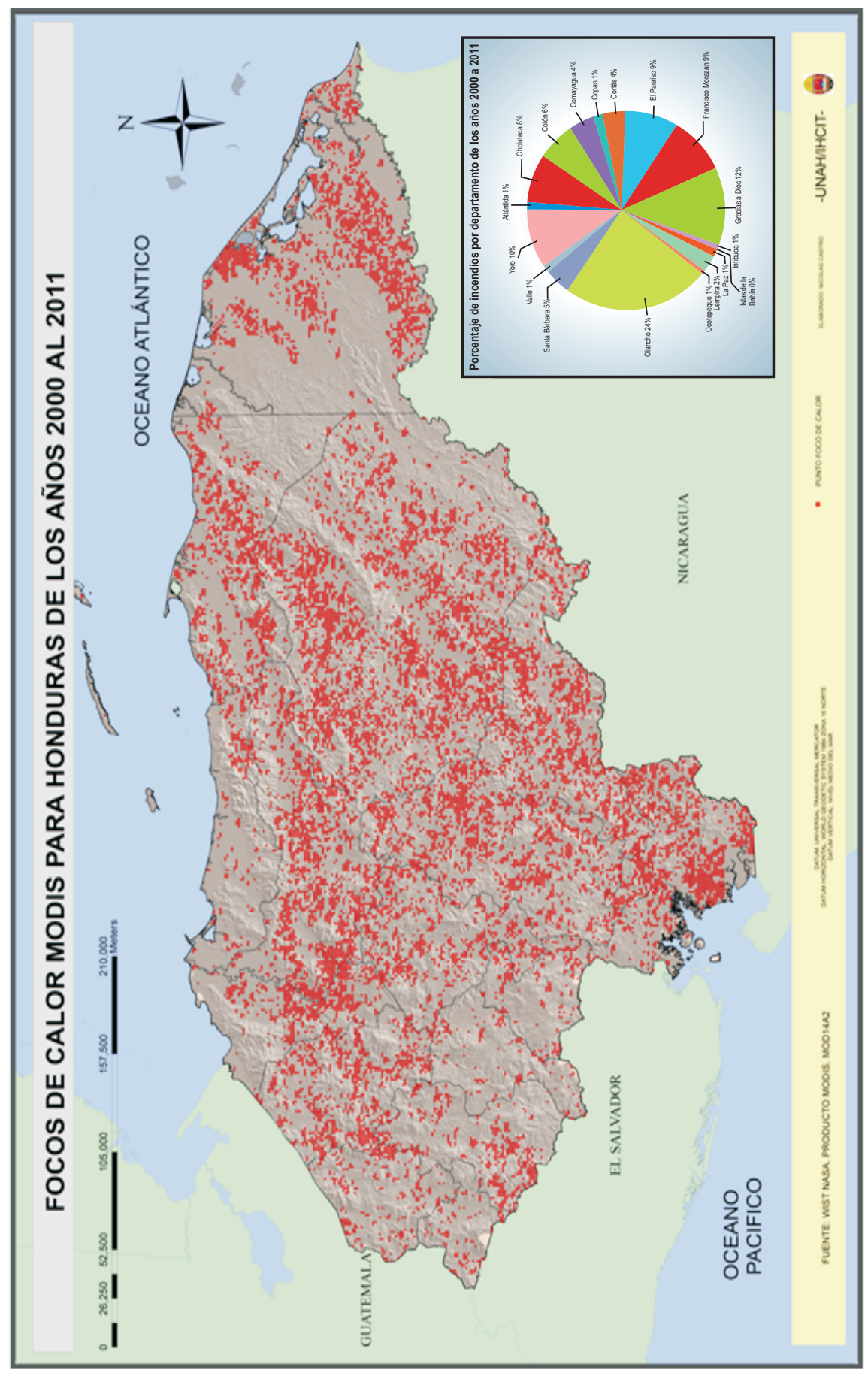


Periodicidad y tendencia en los incendios

En los gráficos 3 y 4 se presentan los resultados del análisis en la tendencia de ocurrencia de incendios y del periodo que presenta el fenómeno para el territorio nacional y para cada departamento. En el gráfico 3 se muestran dos líneas. Una se refiere a los incendios versus tiempo, línea dede puntos rojos, la cual representa a los incendios ocurridos, equiespaciados en tiempo mensual, unidos por una línea azul para hacer notar la secuencia; la línea verde es la tendencia en la ocurrencia de los incendios.

Gráfico 3. Incendios mensuales en Honduras, 2000-2011

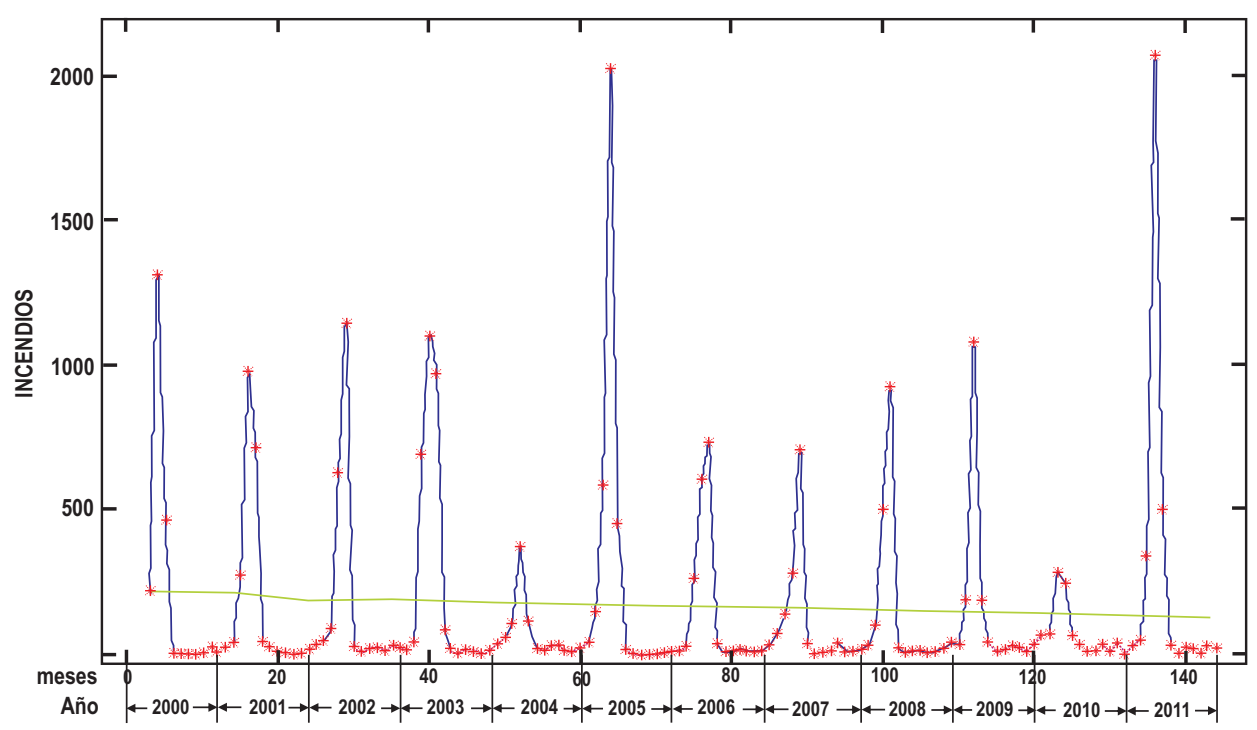

Fuente: Elaboración propia.

Según se observa en el gráfico 3, sobre la naturaleza periódica de los incendios, se puede apreciar que los picos están en marzo, abril y mayo; igualmente, que los años con más ocurrencia de incendios fueron 2005 y 2011. La recta de tendencia, en verde, indica una muy leve tendencia negativa con una pendiente de -0.55 , lo que representa una disminución de un incendio por cada año y diez meses. También se ve que, anteriormente a los años con más incendios, se presentan los años con menos cantidad de incendios, lo que podría significar la existencia de una tendencia más general. 
En el gráfico 4 se muestra la periodicidad en meses versus la potencia de periodo, en el cual es evidente cuáles son los periodos en los que ocurren los incendios y de estos se toma como periodo natural el de mayor potencia.

Gráfico 4. Periodo natural de 12 y 6 meses

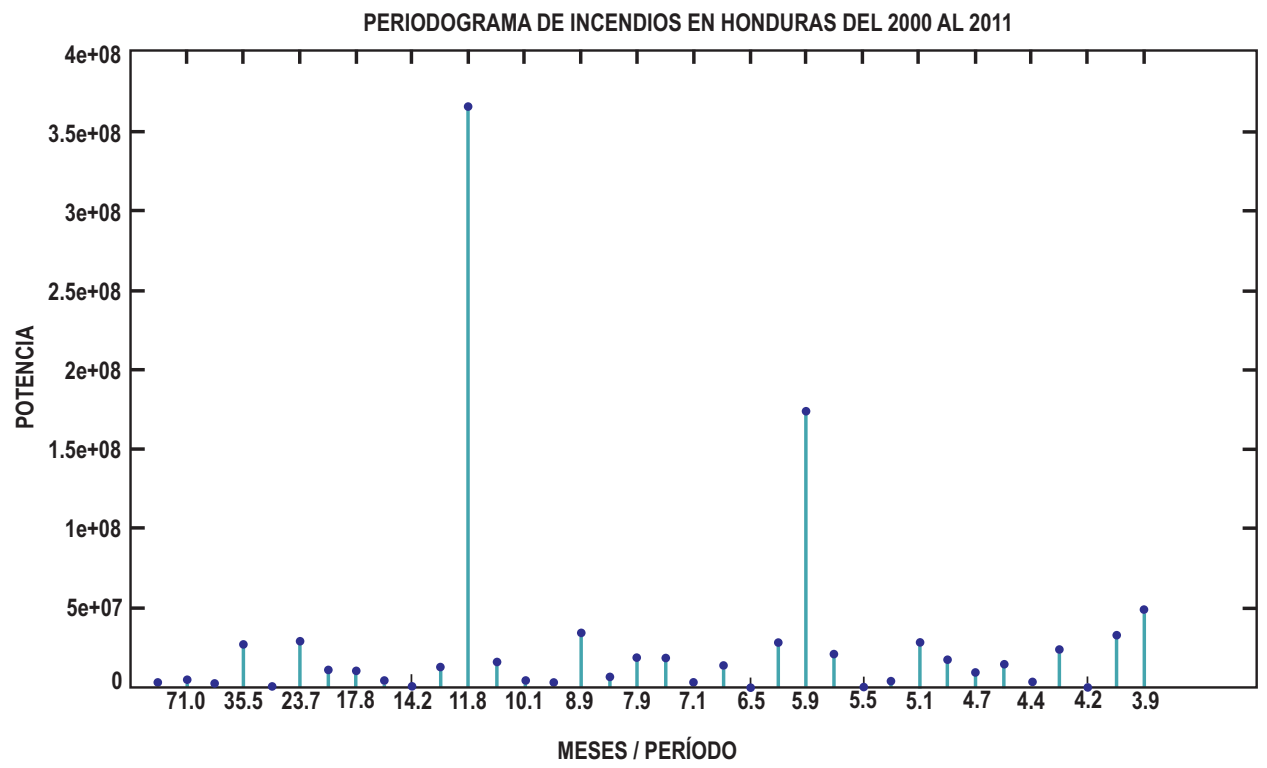

Fuente: Elaboración propia.

El periodo más potente es el de doce meses, lo cual no es extraño, ya que los incendios son un fenómeno de temporada seca y caliente, lo que sucede una vez al año. El siguiente periodo es de seis meses y los demás son más el resultado de la aleatoriedad.

Este mismo procedimiento se utilizó para analizar los departamentos de Honduras por separado, que no está en los gráficos por cuestiones de espacio, pero en el cuadro 14 aparece la pendiente de la recta de comportamiento de cada departamento, además del periodo natural; en el segundo periodo se presentan los incendios en cada departamento. Solamente Colón y Cortés mostraron aumento de incendios. 
Cuadro 14. Pendiente de la recta de comportamiento por departamento y periodo natural

\begin{tabular}{l|c|l|r|}
\hline Departamento & Pendiente & \multicolumn{1}{|c|}{ Comportamiento } & Periodo \\
\hline Atlántida & -0.0098 & Disminución de un incendio cada 8.5 años & 12 meses \\
\hline Choluteca & -0.0376 & Disminución de un incendio cada 2 años & 13 meses \\
\hline Colón & 0.0103 & Disminución de un incendio cada 8 años & 14 meses \\
\hline Comayagua & 0.0356 & Disminución de un incendio cada 2 años & 15 meses \\
\hline Copán & -0.0109 & Disminución de un incendio cada 7.6 años & 16 meses \\
\hline Cortés & 0.0041 & Constante & 17 meses \\
\hline El Paraíso & -0.0445 & Disminución de un incendio cada 2 años & 18 meses \\
\hline Fco. Morazán & -0.0619 & Disminución de un incendio cada 1.3 años & 19 meses \\
\hline Gracias a Dios & -0.0646 & Disminución de un incendio cada 1.3 años & 20 meses \\
\hline Intibucá & -0.0065 & Constante & 21 meses \\
\hline Islas de la Bahía & $-2.12 \mathrm{E}-04$ & No estimable & 22 meses \\
\hline La Paz & -0.0178 & Disminución de un incendio cada 4.7 años & 23 meses \\
\hline Lempira & -0.0046 & Disminución de un incendio cada 2 años & 24 meses \\
\hline Ocotepeque & -0.0068 & Constante & 25 meses \\
\hline Olancho & -0.145 & Disminución de un incendio cada 7 meses & 26 meses \\
Santa Bárbara & -0.0278 & Disminución de un incendio cada 3 años & 27 meses \\
\hline Valle & -0.0049 & Constante & 28 meses \\
Yoro & -0.0514 & Disminución de un incendio cada 1.6 años & 29 meses \\
\hline
\end{tabular}

\section{CONCLUSIONES}

Se realizó un amplio y completo inventario de los incendios en Honduras y se construyó la cartografía correspondiente, lo cual era el objetivo principal.

El cruce de puntos de fuego y territorio departamental arrojó información sobre la periodicidad natural del evento, la cual es marcadamente anual en todos los departamentos, mostrando en todos un segundo periodo de seis meses. Los demás periodos encontrados sí difieren, pero debido a la relativa aleatoriedad.

Con respecto a la tendencia en incendios, esta es un poco menos constante, ya que difiere para cada departamento, aunque es notorio que hay una ligera tendencia decreciente en ellos; excepto en Cortés y Colón, en los que se calculó un pequeño incremento. 


\section{AGRADECIMIENTOS}

A Manuel Rodríguez Maradiaga, jefe del Departamento de Física, por su colaboración y gentileza. Asimismo, a Klaus Wise, por su ayuda en el desarrollo de este trabajo.

\section{BIBLIOGRAFÍA}

Christopher, Justice y Giglio, Louis. (2006). Algorithm technical background document. Recuperado de:

http://modis.gsfc.nasa.gov/data/atbd/atbd_mod14.pdf

Chuvieco Salinero, Emilio. (2008). Teledetección ambiental. Barcelona:Ariel.

Yoram J. Kaufman y Chris Justice. (1998). Monitoring global fires from EOS-MODIS.

Recuperado de: http://modis-atmos.gsfc.nasa.gov/reference/docs/

Kaufman_er_al_\%281998c\%29.pdf 